International Electronic Journal of Algebra

Volume 30 (2021) 269-284

DOI: $10.24330 /$ ieja.969935

\title{
ON THE GORENSTEIN PROPERTY OF THE EHRHART RING OF THE STABLE SET POLYTOPE OF AN H-PERFECT GRAPH
}

\author{
Mitsuhiro Miyazaki \\ Received: 3 September 2020; Accepted: 3 March 2021 \\ Communicated by Abdullah Harmancı
}

\begin{abstract}
In this paper, we give a criterion of the Gorenstein property of the Ehrhart ring of the stable set polytope of an h-perfect graph: the Ehrhart ring of the stable set polytope of an h-perfect graph $G$ is Gorenstein if and only if (1) sizes of maximal cliques are constant (say $n$ ) and (2) (a) $n=1$, (b) $n=2$ and there is no odd cycle without chord and length at least 7 or (c) $n \geq 3$ and there is no odd cycle without chord and length at least 5 .
\end{abstract}

Mathematics Subject Classification (2020): 13H10, 52B20, 13C14

Keywords: Gorenstein ring, h-perfect graph, Ehrhart ring, stable set polytope

\section{Introduction}

Recently, Hibi and Tsuchiya [5] showed that the Ehrhart ring of the stable set polytope of an odd cycle graph is Gorenstein if and only if the length of the cycle is less than or equal to 5 . They used the fact that a cycle graph is t-perfect. On the other hand, Ohsugi and Hibi [8] showed that the Ehrhart ring of the stable set polytope of a perfect graph is Gorenstein if and only if all maximal cliques have the same size.

Meanwhile, Sbihi and Uhry [9] introduced the notion of h-perfect graphs as a common generalization of perfect and t-perfect graphs: a graph is h-perfect if its stable set polytope is defined by the constraints nonnegativity of vertices and the constraints corresponding to cliques and odd cycles.

In this paper, we characterize Gorenstein property of the Ehrhart ring of the stable set polytope of an h-perfect graph by using the trace of the canonical module of a Cohen-Macaulay ring. This result is a generalization of both results of OhsugiHibi and Hibi-Tsuchiya. 


\section{Preliminaries}

In this section, we establish notation and terminology. In this paper, all rings and algebras are assumed to be commutative with an identity element unless stated otherwise. Further, all graphs are finite simple graphs without loop. We denote the set of nonnegative integers, the set of integers, the set of rational numbers and the set of real numbers by $\mathbb{N}, \mathbb{Z}, \mathbb{Q}$ and $\mathbb{R}$ respectively. For a set $X$, we denote by $\# X$ the cardinality of $X$. For sets $X$ and $Y$, we define $X \backslash Y:=\{x \in X \mid x \notin Y\}$. For nonempty sets $X$ and $Y$, we denote the set of maps from $X$ to $Y$ by $Y^{X}$. If $X$ is a finite set, we identify $\mathbb{R}^{X}$ with the Euclidean space $\mathbb{R}^{\# X}$. For $f, f_{1}, f_{2} \in \mathbb{R}^{X}$ and $a \in \mathbb{R}$, we define maps $f_{1} \pm f_{2}$ and af by $\left(f_{1} \pm f_{2}\right)(x)=f_{1}(x) \pm f_{2}(x)$ and $(a f)(x)=a(f(x))$ for $x \in X$. We denote the zero map $X \ni x \mapsto 0 \in \mathbb{R}$ by 0 . Let $A$ be a subset of $X$. We define the characteristic function $\chi_{A} \in \mathbb{R}^{X}$ by $\chi_{A}(x)=1$ for $x \in A$ and $\chi_{A}(x)=0$ for $x \in X \backslash A$. For a nonempty subset $\mathscr{X}$ of $\mathbb{R}^{X}$, we denote by conv $\mathscr{X}$ (resp. aff $\mathscr{X}$ ) the convex hull (resp. affine span) of $\mathscr{X}$.

Definition 2.1. Let $X$ be a finite set and $\xi \in \mathbb{R}^{X}$. For $B \subset X$, we set $\xi^{+}(B):=$ $\sum_{b \in B} \xi(b)$. We define the empty sum to be 0 , i.e., if $B=\emptyset$, then $\xi^{+}(B)=0$.

A stable set of a graph $G=(V, E)$ is a subset $S$ of $V$ with no two elements of $S$ are adjacent. We treat the empty set as a stable set.

Definition 2.2. The stable set polytope $\operatorname{STAB}(G)$ of a graph $G=(V, E)$ is

$$
\operatorname{conv}\left\{\chi_{S} \in \mathbb{R}^{V} \mid S \text { is a stable set of } G \text {. }\right\}
$$

It is clear that for $f \in \operatorname{STAB}(G)$,

(1) $f(x) \geq 0$ for any $x \in V$.

(2) $f^{+}(K) \leq 1$ for any clique $K$ in $G$.

(3) $f^{+}(C) \leq \frac{\# C-1}{2}$ for any odd cycle $C$.

Definition 2.3. We set

$$
\operatorname{HSTAB}(G):=\left\{f \in \mathbb{R}^{V} \mid f \text { satisfies (1), (2) and (3) above }\right\} .
$$

If $\operatorname{HSTAB}(G)=\operatorname{STAB}(G)$, then we say that $G$ is an h-perfect graph.

It is immediately seen that $\operatorname{STAB}(G) \subset \operatorname{HSTAB}(G)$ for a general graph $G$. Note that $\operatorname{HSTAB}(G)$ is a convex polytope since it is bounded by (1) and (2).

We fix notation about Ehrhart rings. Let $\mathbb{K}$ be a field, $X$ a finite set and $\mathscr{P}$ a rational convex polytope in $\mathbb{R}^{X}$, i.e., a convex polytope whose vertices are contained in $\mathbb{Q}^{X}$. Let $-\infty$ be a new element with $-\infty \notin X$ and set $X^{-}:=X \cup\{-\infty\}$. Also 
let $\left\{T_{x}\right\}_{x \in X^{-}}$be a family of indeterminates indexed by $X^{-}$. For $f \in \mathbb{Z}^{X^{-}}$, we denote the Laurent monomial $\prod_{x \in X^{-}} T_{x}^{f(x)}$ by $T^{f}$. We set $\operatorname{deg} T_{x}=0$ for $x \in X$ and $\operatorname{deg} T_{-\infty}=1$. Then the Ehrhart ring of $\mathscr{P}$ over a field $\mathbb{K}$ is the $\mathbb{N}$-graded subring

$$
\mathbb{K}\left[T^{f}\left|f \in \mathbb{Z}^{X^{-}}, f(-\infty)>0, \frac{1}{f(-\infty)} f\right|_{X} \in \mathscr{P}\right]
$$

of the Laurent polynomial ring $\mathbb{K}\left[T_{x}^{ \pm 1} \mid x \in X\right]\left[T_{-\infty}\right]$, where $\left.f\right|_{X}$ is the restriction of $f$ to $X$. We denote the Ehrhart ring of $\mathscr{P}$ over $\mathbb{K}$ by $E_{\mathbb{K}}[\mathscr{P}]$.

It is known that $E_{\mathbb{K}}[\mathscr{P}]$ is Noetherian. Therefore normal and Cohen-Macaulay by the criterion of Hochster [6]. Further, by the description of the canonical module of a normal affine semigroup ring by Stanley [10, p. 82], we see the following.

Lemma 2.4. The ideal

$$
\bigoplus_{f \in \mathbb{Z}^{X^{-}}, f(-\infty)>0,\left.\frac{1}{f(-\infty)} f\right|_{X} \in \operatorname{relint} \mathscr{P}} \mathbb{K} T^{f}
$$

of $E_{\mathbb{K}}[\mathscr{P}]$ is the canonical module of $E_{\mathbb{K}}[\mathscr{P}]$, where relint $\mathscr{P}$ denotes the interior of $\mathscr{P}$ in the topological space aff $\mathscr{P}$.

We denote the ideal of the above lemma by $\omega_{E_{\mathbb{K}}[\mathscr{P}]}$ and call the canonical ideal of $E_{\mathbb{K}}[\mathscr{P}]$.

Let $R$ be a Noetherian normal domain. Then the set of divisorial ideals $\operatorname{Div}(R)$ form a group by the operation $I \cdot J:=R:_{Q(R)}\left(R:_{Q(R)} I J\right)$ for $I, J \in \operatorname{Div}(R)$, where $Q(R)$ is the quotient field of $R$. See e.g., [3, Chapter I] for details. We denote the $n$-th power of $I \in \operatorname{Div}(R)$ in this group by $I^{(n)}$. Note that if $R$ is a Cohen-Macaulay local or graded ring over a field with canonical module $\omega$, then $\omega$ is isomorphic to a divisorial ideal. See e.g., [1, Chapter 3] for details.

\section{The trace of the canonical module and Gorenstein property}

In this section, we give a criterion of Gorenstein property of the Ehrhart ring of $\operatorname{HSTAB}(G)$ of a graph $G$. As a consequence, we give a criterion of Gorenstein property of the Ehrhart ring of the stable set polytope of an h-perfect graph. First we recall the following.

Definition 3.1. Let $R$ be a ring and $M$ an $R$-module. We set

$$
\operatorname{tr}(M):=\sum_{\varphi \in \operatorname{Hom}(M, R)} \varphi(M)
$$

and call $\operatorname{tr}(M)$ the trace of $M$.

Next we recall the following basic fact on the trace of an ideal. 
Fact 3.2 ([4, Lemma 1.1]). Let $R$ be a Noetherian ring and $I$ an ideal of $R$ with grade $I>0$. Also let $Q(R)$ be the total quotient ring of fractions of $R$ and set $I^{-1}:=\{x \in Q(R) \mid x I \subset R\}$. Then

$$
\operatorname{tr}(I)=I^{-1} I
$$

Note that if $R$ is a domain and $I$ is a divisorial ideal, then $I^{-1}=I^{(-1)}$. Moreover, we recall the following.

Fact 3.3 ([4, Lemma 2.1]). Let $R$ be a Cohen-Macaulay local or graded ring over a field with canonical module $\omega_{R}$. Then for $\mathfrak{p} \in \operatorname{Spec}(R)$,

$$
R_{\mathfrak{p}} \text { is Gorenstein } \Longleftrightarrow \mathfrak{p} \not \supset \operatorname{tr}\left(\omega_{R}\right) \text {. }
$$

In particular, $R$ is Gorenstein if and only if $\operatorname{tr}\left(\omega_{R}\right) \ni 1$.

In the rest of this paper, we fix a graph $G=(V, E)$. First we note the following.

Lemma 3.4. Let $f \in \mathbb{R}^{V}$. Then $f \in \operatorname{HSTAB}(G)$ if and only if

(1) $f(x) \geq 0$ for any $x \in V$,

(2) $f^{+}(K) \leq 1$ for any maximal clique $K$ of $G$ and

(3) $f^{+}(C) \leq \frac{\# C-1}{2}$ for any odd cycle $C$ without chord with length at least 5 .

In order to make statements simple, we make the following.

Definition 3.5. Let $n \in \mathbb{Z}$. We set $\mathcal{U}^{(n)}:=\left\{\mu \in \mathbb{Z}^{V^{-}} \mid \mu(z) \geq n\right.$ for any $z \in V$, $\mu^{+}(K) \leq \mu(-\infty)-n$ for any maximal clique $K$ of $G$ and $\mu^{+}(C) \leq \mu(-\infty) \frac{\# C-1}{2}-n$ for any odd cycle $C$ without chord and length at least 5$\}$.

Note that if $\mu \in \mathcal{U}^{(n)}$ and $\mu^{\prime} \in \mathcal{U}^{(m)}$, then $\mu+\mu^{\prime} \in \mathcal{U}^{(n+m)}$. By Lemmas 2.4, 3.4 and Definition 3.5, we see the following.

Corollary 3.6. Let $\mu \in \mathbb{Z}^{V^{-}}$. Then $T^{\mu} \in E_{\mathbb{K}}[\operatorname{HSTAB}(G)]$ if and only if $\mu \in \mathcal{U}^{(0)}$. Moreover, $T^{\mu} \in \omega_{E_{\mathbb{K}}[\operatorname{HSTAB}(G)]}$ if and only if $\mu \in \mathcal{U}^{(1)}$.

Next we show the following.

Proposition 3.7. Let $n \in \mathbb{Z}$ and $\mu \in \mathbb{Z}^{V^{-}}$and set $\omega:=\omega_{E_{\mathbb{K}}[\operatorname{HSTAB}(G)]}$. Then $T^{\mu} \in \omega^{(n)}$ if and only if $\mu \in \mathcal{U}^{(n)}$.

Proof. The cases where $n=0$ and $n=1$ are stated in Corollary 3.6. Next, we consider the case where $n<0$. Set $m=-n$. First we show the "if" part of this case. Let $\mu_{1}, \ldots, \mu_{m}$ be arbitrary elements of $\mathcal{U}^{(1)}$. Then, since $\mu \in \mathcal{U}^{(n)}$, we see that $\mu+\mu_{1}+\cdots+\mu_{m} \in \mathcal{U}^{(0)}$. Therefore, by Corollary 3.6, we see that $T^{\mu} T^{\mu_{1}} \cdots T^{\mu_{m}} \in$ 
$E_{\mathbb{K}}[\operatorname{HSTAB}(G)]$. Since $T^{\mu_{1}}, \ldots, T^{\mu_{m}}$ are arbitrary Laurent monomials in $\omega$ by Corollary 3.6 , we see that

$$
T^{\mu} \in E_{\mathbb{K}}[\operatorname{HSTAB}(G)]: \omega^{m}=\omega^{(n)} .
$$

Next, we show the "only if" part of this case. Let $N$ be a huge integer $(N>\# V)$. First let $\mu_{1}$ be an element of $\mathbb{Z}^{V^{-}}$with

$$
\mu_{1}(z):= \begin{cases}1 & z \in V \\ N & z=-\infty\end{cases}
$$

Then it is easily seen that $\mu_{1} \in \mathcal{U}^{(1)}$. Thus, $T^{\mu_{1}} \in \omega$ by Corollary 3.6. Since $T^{\mu} \in$ $\omega^{(n)}=E_{\mathbb{K}}[\operatorname{HSTAB}(G)]: \omega^{m}$, we see that $T^{\mu+m \mu_{1}}=T^{\mu}\left(T^{\mu_{1}}\right)^{m} \in E_{\mathbb{K}}[\operatorname{HSTAB}(G)]$. Thus, by Corollary 3.6, we see that $\left(\mu+m \mu_{1}\right)(z) \geq 0$ and therefore

$$
\mu(z) \geq-m=n \text { for any } z \in V .
$$

Next, let $K$ be an arbitrary maximal clique of $G$. Set

$$
\mu_{2}(z):= \begin{cases}N & z \in K, \\ 1 & z \in V \backslash K, \\ (\# K) N+1 & z=-\infty .\end{cases}
$$

We will show that $\mu_{2} \in \mathcal{U}^{(1)}$. First $\mu_{2}(z) \geq 1$ for any $z \in V$ by the definition of $\mu_{2}$. Moreover, let $K^{\prime}$ be an arbitrary maximal clique of $G$. If $K^{\prime}=K$, then $\mu_{2}^{+}\left(K^{\prime}\right)=\mu_{2}^{+}(K)=(\# K) N=\mu_{2}(-\infty)-1$. If $K^{\prime} \neq K$, then since $\#\left(K^{\prime} \cap K\right) \leq$ $\# K-1, \mu_{2}^{+}\left(K^{\prime}\right)<(\# K-1) N+\# V<(\# K) N=\mu_{2}(-\infty)-1$. Further, let $C$ be an arbitrary odd cycle without chord and length at least 5 . If $K \cap C=\emptyset$, then $\mu_{2}^{+}(C) \leq \# V \leq N \frac{\# C-1}{2}-1 \leq \mu_{2}(-\infty) \frac{\# C-1}{2}-1$. Suppose that $K \cap C \neq \emptyset$. Then $\#(K \cap C) \leq 2$, since $C$ does not have a chord, and $\# K \geq 2$, since $K$ is a maximal clique. Thus,

$$
\begin{aligned}
\mu_{2}^{+}(C) & \leq 2 N+\# V \\
& \leq 3 N-1 \\
& \leq 2 N \frac{\# C-1}{2}-1 \\
& \leq \mu_{2}(-\infty) \frac{\# C-1}{2}-1 .
\end{aligned}
$$

Therefore, $\mu_{2} \in \mathcal{U}^{(1)}$ and we see by Corollary 3.6 that $T^{\mu_{2}} \in \omega$. Since $T^{\mu} \in$ $\omega^{(n)}=E_{\mathbb{K}}[\operatorname{HSTAB}(G)]: \omega^{m}$, we see that $T^{\mu+m \mu_{2}}=T^{\mu}\left(T^{\mu_{2}}\right)^{m} \in E_{\mathbb{K}}[\operatorname{HSTAB}(G)]$ and therefore, $\mu+m \mu_{2} \in \mathcal{U}^{(0)}$. Thus, $\left(\mu+m \mu_{2}\right)^{+}(K) \leq\left(\mu+m \mu_{2}\right)(-\infty)$. Since $\mu_{2}^{+}(K)=(\# K) N$ and $\mu_{2}(-\infty)=(\# K) N+1$, we see that

$$
\mu^{+}(K) \leq \mu(-\infty)+m=\mu(-\infty)-n .
$$


Next, let $C$ be an arbitrary odd cycle without chord and length at least 5 . Take $c_{0} \in C$ and set

$$
\mu_{3}(z):= \begin{cases}N(\# C-1) & z \in C \backslash\left\{c_{0}\right\}, \\ N(\# C-1)-1 & z=c_{0}, \\ 1 & z \in V \backslash C, \\ 2 N(\# C) & z=-\infty .\end{cases}
$$

We will show that $\mu_{3} \in \mathcal{U}^{(1)}$. It is clear that $\mu_{3}(z) \geq 1$ for any $z \in V$. Let $K$ be an arbitrary maximal clique of $G$. Then $\#(K \cap C) \leq 2$ and therefore

$$
\mu_{3}^{+}(K) \leq 2 N(\# C-1)+\# V \leq 2 N(\# C)-1=\mu_{3}(-\infty)-1 .
$$

Let $C^{\prime}$ be an arbitrary odd cycle without chord and length at least 5 . If $C^{\prime}=C$, then

$$
\begin{aligned}
\mu_{3}^{+}\left(C^{\prime}\right) & =\mu_{3}^{+}(C) \\
& =(\# C) N(\# C-1)-1 \\
& =2 N(\# C) \frac{\# C-1}{2}-1 \\
& =\mu_{3}(-\infty) \frac{\# C^{\prime}-1}{2}-1 .
\end{aligned}
$$

Suppose that $C^{\prime} \neq C$. Then $\#\left(C \cap C^{\prime}\right) \leq \# C^{\prime}-1$, since $C$ does not have a chord. Therefore,

$$
\begin{aligned}
\mu_{3}^{+}\left(C^{\prime}\right) & \leq\left(\# C^{\prime}-1\right) N(\# C-1)+\# V \\
& \leq N(\# C)\left(\# C^{\prime}-1\right)-1 \\
& =2 N(\# C) \frac{\# C^{\prime}-1}{2}-1 \\
& =\mu_{3}(-\infty) \frac{\# C^{\prime}-1}{2}-1 .
\end{aligned}
$$

Thus, we see that $\mu_{3} \in \mathcal{U}^{(1)}$ and by Corollary 3.6 that $T^{\mu_{3}} \in \omega$. Since $T^{\mu} \in \omega^{(n)}=$ $E_{\mathbb{K}}[\operatorname{HSTAB}(G)]: \omega^{m}$, we see that $T^{\mu+m \mu_{3}}=T^{\mu}\left(T^{\mu_{3}}\right)^{m} \in E_{\mathbb{K}}[\operatorname{HSTAB}(G)]$ and therefore $\mu+m \mu_{3} \in \mathcal{U}^{(0)}$. Thus,

$$
\left(\mu+m \mu_{3}\right)^{+}(C) \leq\left(\mu+m \mu_{3}\right)(-\infty) \frac{\# C-1}{2} .
$$

Since $\mu_{3}^{+}(C)=\mu_{3}(-\infty) \frac{\# C-1}{2}-1$ by equation (3.1), we see that

$$
\mu^{+}(C) \leq \mu(-\infty) \frac{\# C-1}{2}+m=\mu(-\infty) \frac{\# C-1}{2}-n .
$$

Thus, we see that $\mu \in \mathcal{U}^{(n)}$. 
Next we consider the case where $n \geq 2$. If $\mu \in \mathcal{U}^{(n)}$, then for any $\mu^{\prime} \in \mathcal{U}^{(-n)}$, $\mu+\mu^{\prime} \in \mathcal{U}^{(0)}$ by the fact noted after Definition 3.5. Since $\omega^{(-n)}$ is the $\mathbb{K}$-vector space with basis $T^{\mu^{\prime}}$ with $\mu^{\prime} \in \mathcal{U}^{(-n)}$ by the case shown above, we see that $T^{\mu} \in$ $E_{\mathbb{K}}[\operatorname{HSTAB}(G)]: \omega^{(-n)}=\omega^{(n)}$.

Conversely, assume that $T^{\mu} \in \omega^{(n)}$. Set

$$
\mu_{4}(z):= \begin{cases}-1 & z \in V, \\ 0 & z=-\infty .\end{cases}
$$

Then it is easily verified that $\mu_{4} \in \mathcal{U}^{(-1)}$ and therefore by the fact shown above, we see that $T^{\mu_{4}} \in \omega^{(-1)}$. Therefore, $T^{\mu+n \mu_{4}}=T^{\mu}\left(T^{\mu_{4}}\right)^{n} \in E_{\mathbb{K}}[\operatorname{HSTAB}(G)]$ and we see by Corollary 3.6 that $\mu+n \mu_{4} \in \mathcal{U}^{(0)}$. Thus, $\left(\mu+n \mu_{4}\right)(z) \geq 0$ for any $z \in V$ and we see that

$$
\mu(z) \geq n
$$

for any $z \in V$. Next, let $K$ be an arbitrary maximal clique of $G$. Take $k_{0} \in K$ and set

$$
\mu_{5}(z):= \begin{cases}0 & z \in\left(K \backslash\left\{k_{0}\right\}\right) \cup\{-\infty\}, \\ 1 & z=k_{0}, \\ -1 & z \in V \backslash K .\end{cases}
$$

Then, it is easily verified that $\mu_{5} \in \mathcal{U}^{(-1)}$ and $\mu_{5}^{+}(K)=1$. In particular, $T^{\mu_{5}} \in$ $\omega^{(-1)}$. Since $\mu+n \mu_{5} \in \mathcal{U}^{(0)}$ by the same reason as above, we see that

$$
\left(\mu+n \mu_{5}\right)^{+}(K) \leq \mu(-\infty)+n \mu_{5}(-\infty)=\mu(-\infty) .
$$

Therefore,

$$
\mu^{+}(K) \leq \mu(-\infty)-n,
$$

since $\mu_{5}^{+}(K)=1$. Finally, let $C$ be an arbitrary odd cycle without chord and length at least 5 . Take $c_{0} \in C$ and set

$$
\mu_{6}(z):= \begin{cases}0 & z \in\left(C \backslash\left\{c_{0}\right\}\right) \cup\{-\infty\}, \\ 1 & z=c_{0} \\ -1 & z \in V \backslash C .\end{cases}
$$

Then, it is easily verified that $\mu_{6} \in \mathcal{U}^{(-1)}$ and $\mu_{6}^{+}(C)=1$. In particular, $T^{\mu_{6}} \in$ $\omega^{(-1)}$. Thus, $\mu+n \mu_{6} \in \mathcal{U}^{(0)}$ and since

$$
\left(\mu+n \mu_{6}\right)^{+}(C) \leq\left(\mu+n \mu_{6}\right)(-\infty) \frac{\# C-1}{2}=\mu(-\infty) \frac{\# C-1}{2},
$$

we see that

$$
\mu^{+}(C) \leq \mu(-\infty) \frac{\# C-1}{2}-n,
$$

since $\mu_{6}^{+}(C)=1$. Therefore, $\mu \in \mathcal{U}^{(n)}$. 
Now we show the following.

Theorem 3.8. $E_{\mathbb{K}}[\operatorname{HSTAB}(G)]$ is Gorenstein if and only if

(1) Sizes of maximal cliques are constant (say $n$ ) and

(2) (a) $n=1$,

(b) $n=2$ and there is no odd cycle without chord and length at least 7 or

(c) $n \geq 3$ and there is no odd cycle without chord and length at least 5 .

In particular, if $G$ is an h-perfect graph, then $E_{\mathbb{K}}[\operatorname{STAB}(G)]$ is Gorenstein if and only if (1) and (2) above are satisfied.

Proof. We denote $\omega_{E_{\mathbb{K}}[\operatorname{HSTAB}(G)]}$ by $\omega$. We first prove the "if" part. Set $\eta(v)=1$, $\zeta(v)=-1$ for $v \in V, \eta(-\infty)=n+1$ and $\zeta(-\infty)=-n-1$. We will show that $\eta \in \mathcal{U}^{(1)}$ and $\zeta \in \mathcal{U}^{(-1)}$.

First, it is clear from the definition that $\eta(v) \geq 1$ and $\zeta(v) \geq-1$ for any $v \in V$ and $\eta^{+}(K) \leq \eta(-\infty)-1$ and $\zeta^{+}(K) \leq \zeta(-\infty)+1$ for any maximal clique $K$ of $G$. If (2)(a) is satisfied, then $E=\emptyset$ and there is no odd cycle. Thus, we see that $\eta \in \mathcal{U}^{(1)}$ and $\zeta \in \mathcal{U}^{(-1)}$ by the trivial reason. We also see that if (2)(c) is satisfied, then $\eta \in \mathcal{U}^{(1)}$ and $\zeta \in \mathcal{U}^{(-1)}$ by the trivial reason. Now suppose that $(2)(\mathrm{b})$ is satisfied. If $C$ is an odd cycle without chord and length at least 5 , then the length of $C$ is 5 by the assumption. Since $\eta(v)=1(\operatorname{resp} . \zeta(v)=-1)$ for any $v \in V$ and $\eta(-\infty)=3($ resp. $\zeta(-\infty)=-3)$, we see that

$$
\eta^{+}(C)=5=3 \cdot \frac{5-1}{2}-1=\eta(-\infty) \frac{\# C-1}{2}-1
$$

(resp. $\left.\zeta^{+}(C)=-5=-3 \cdot \frac{5-1}{2}+1=\eta(-\infty) \frac{\# C-1}{2}+1\right)$. Therefore, $\eta \in \mathcal{U}^{(1)}$ and $\zeta \in \mathcal{U}^{(-1)}$.

Since $\eta+\zeta=0$, we see by Proposition 3.7 and Fact 3.2 that

$$
1=T^{0} \in \omega^{-1} \omega=\operatorname{tr}(\omega)
$$

Thus, we see by Fact 3.3 that $E_{\mathbb{K}}[\operatorname{HSTAB}(G)]$ is Gorenstein.

Now we prove the "only if" part. By Facts 3.2 and 3.3, we see that

$$
1 \in \omega^{-1} \omega=\omega^{(-1)} \omega
$$

Therefore, by Proposition 3.7, we see that there are $\eta \in \mathcal{U}^{(1)}$ and $\zeta \in \mathcal{U}^{(-1)}$ with $\eta+\zeta=0$. Let $v$ be an arbitrary element of $V$. Then, since $\eta(v) \geq 1$ and $\zeta(v) \geq-1$ and $(\eta+\zeta)(v)=0$, we see that $\eta(v)=1$ and $\zeta(v)=-1$. Let $K$ and $K^{\prime}$ be arbitrary 
maximal cliques of $G$. If $\# K>\# K^{\prime}$, then

$$
\begin{aligned}
& \eta(-\infty) \geq \eta^{+}(K)+1=\# K+1, \\
& \zeta(-\infty) \geq \zeta^{+}\left(K^{\prime}\right)-1=-\left(\# K^{\prime}\right)-1
\end{aligned}
$$

and therefore,

$$
(\eta+\zeta)(-\infty) \geq \# K-\left(\# K^{\prime}\right)>0 .
$$

This contradicts to $\eta+\zeta=0$. Therefore $\# K \leq \# K^{\prime}$ and by symmetry, we see that $\# K=\# K^{\prime}$. Since $K$ and $K^{\prime}$ are arbitrary maximal cliques of $G$, we see that the size of maximal cliques of $G$ are constant.

Let $n$ be this constant size. Then since

$$
\eta(-\infty) \geq n+1, \quad \zeta(-\infty) \geq-n-1 \quad \text { and } \quad(\eta+\zeta)(-\infty)=0,
$$

we see that

$$
\eta(-\infty)=n+1 \quad \text { and } \quad \zeta(-\infty)=-n-1 .
$$

Suppose that there is an odd cycle $C$ without chord and length at least 5 . Then $n \geq 2$,

$$
\eta^{+}(C) \leq \eta(-\infty) \frac{\# C-1}{2}-1 \quad \text { and } \quad \zeta^{+}(C) \leq \zeta(-\infty) \frac{\# C-1}{2}+1 .
$$

Thus, since $\eta^{+}(C)=\# C, \zeta^{+}(C)=-(\# C), \eta(-\infty)=n+1$ and $\zeta(-\infty)=-n-1$, we see that

$$
\frac{2(\# C+1)}{\# C-1}=\frac{2\left(\eta^{+}(C)+1\right)}{\# C-1} \leq \eta(-\infty)=n+1
$$

and

$$
-\frac{2(\# C+1)}{\# C-1}=\frac{2\left(\zeta^{+}(C)-1\right)}{\# C-1} \leq \zeta(-\infty)=-n-1,
$$

i.e.,

$$
n+1 \leq 2+\frac{4}{\# C-1} \leq n+1 .
$$

Therefore, $\# C=5$ and $n=2$, since $n$ is an integer.

Thus, we see that if $n \geq 3$, then there is no odd cycle without chord and length at least 5 . Moreover, we see that if $n=2$, then there is no odd cycle without chord and length at least 7 .

Set

$$
\operatorname{TSTAB}(G):=\left\{\begin{array}{l|l}
f \in \mathbb{R}^{V} & \begin{array}{l}
0 \leq f(x) \leq 1 \text { for any } x \in V, f^{+}(e) \leq 1 \\
\text { for any } e \in E \text { and } f^{+}(C) \leq \frac{\# C-1}{2} \text { for any } \\
\text { odd cycle } C
\end{array}
\end{array}\right\}
$$


and

$$
\operatorname{QSTAB}(G):=\left\{\begin{array}{l|l}
f \in \mathbb{R}^{V} & \begin{array}{l}
f(x) \geq 0 \text { for any } x \in V \text { and } f^{+}(K) \leq 1 \\
\text { for any clique } K \text { in } G
\end{array}
\end{array}\right\} .
$$

If $\operatorname{STAB}(G)=\operatorname{TSTAB}(G)$, then $G$ is called a t-perfect graph. It is easily verified that $\operatorname{HSTAB}(G) \subset \operatorname{TSTAB}(G)$. In particular, t-perfect graphs are h-perfect. Further, by $[2$, Theorem 3.1], $G$ is perfect if and only if $\operatorname{STAB}(G)=\operatorname{QSTAB}(G)$. Since $\operatorname{HSTAB}(G) \subset \operatorname{QSTAB}(G)$ by the definition, perfect graphs are h-perfect.

Corollary 3.9. (1) Suppose that $G$ is t-perfect. Then $E_{\mathbb{K}}[\operatorname{STAB}(G)]$ is Gorenstein if and only if

(a) Sizes of maximal cliques are constant (say $n$ ) and

(b) (i) $n=1$,

(ii) $n=2$ and there is no odd cycle without chord and length at least 7 or

(iii) $n=3$ and there is no odd cycle without chord and length at least 5.

(2) (Ohsugi-Hibi) Suppose that $G$ is perfect. Then $E_{\mathbb{K}}[\operatorname{STAB}(G)]$ is Gorenstein if and only if sizes of maximal cliques are constant.

Proof. (1) Since a t-perfect graph is h-perfect and has no clique with size more than 3 , the result follows from Theorem 3.8.

(2) Since a perfect graph is h-perfect and has no odd cycle without chord and length at least 5 , the result follows from Theorem 3.8.

Remark 3.10. Set $\mathscr{K}:=\{K \subset V \mid K$ is a clique of $G$ and size of $K$ is less than or equal to 3$\}$. Then

$$
\operatorname{TSTAB}(G)=\left\{\begin{array}{l|l}
f \in \mathbb{R}^{V} & \begin{array}{l}
f(x) \geq 0 \text { for any } x \in V, f^{+}(K) \leq 1 \\
\text { for any maximal element } K \text { of } \mathscr{K} \text { and } \\
f^{+}(C) \leq \frac{\# C-1}{2} \text { for any odd cycle } C \text { with- } \\
\text { out chord and length at least } 5
\end{array}
\end{array}\right\} .
$$

By defining $\mathcal{U}^{(n)}:=\left\{\mu \in \mathbb{Z}^{V^{-}} \mid \mu(z) \geq n\right.$ for any $z \in V, \mu^{+}(K) \leq \mu(-\infty)-n$ for any maximal element $K$ of $\mathscr{K}$ and $\mu^{+}(C) \leq \mu(-\infty) \frac{\# C-1}{2}-n$ for any odd cycle $C$ without chord and length at least 5$\}$ for $n \in \mathbb{Z}$, one can verify that $T^{\mu} \in$ $\omega_{E_{\mathbb{K}}[\operatorname{TSTAB}(G)]}^{(n)}$ if and only if $\mu \in \mathfrak{t} \mathcal{U}^{(n)}$ along the same line as the proof of Proposition 3.7. Further, it is verified along the same line as the proof of Theorem 3.8 that $E_{\mathbb{K}}[\operatorname{TSTAB}(G)]$ is Gorenstein if and only if

(1) Sizes of maximal elements of $\mathscr{K}$ are constant (say $n$ ) and

(2) (a) $n=1$, 
(b) $n=2$ and there is no odd cycle without chord and length at least 7 or

(c) $n=3$ and there is no odd cycle without chord and length at least 5,

i.e.,

(1) $E=\emptyset$,

(2) $G$ has no isolated vertex nor triangle and there is no odd cycle without chord and length at least 7 or

(3) all maximal cliques of $G$ have size at least 3 and there is no odd cycle without chord and length at least 5 .

Similarly, by defining $q \mathcal{U}^{(n)}:=\left\{\mu \in \mathbb{Z}^{V^{-}} \mid \mu(z) \geq n\right.$ for any $z \in V$ and $\mu^{+}(K) \leq$ $\mu(-\infty)-n$ for any maximal clique of $G\}$ for $n \in \mathbb{Z}$, one can verify that $T^{\mu} \in$ $\omega_{E_{K}[\operatorname{QSTAB}(G)]}^{(n)}$ if and only if $\mu \in q \mathcal{U}^{(n)}$ along the same line as the proof of Proposition 3.7. Further, it is verified along the same line as the proof of Theorem 3.8 that $E_{\mathbb{K}}[\operatorname{QSTAB}(G)]$ is Gorenstein if and only if sizes of maximal cliques are constant.

A graph $G=(V, E)$ is called a comparability graph if $V$ is a partially ordered set (poset for short) and $E=\{\{a, b\} \mid a<b$ or $b<a$ in $V\}$. If $G$ is a comparability graph, then $G$ is perfect and $\operatorname{STAB}(G), \operatorname{QSTAB}(G)$ and $\operatorname{HSTAB}(G)$ coincide with the chain polytope of $V$ (cf. [11]). We showed that the symbolic powers of the canonical ideal of the Ehrhart ring of the chain polytope of a poset coincide with the ordinary powers [7, Theorem 3.8].

Unlike the case of the chain polytope of a poset, the symbolic power of the canonical ideal of the Ehrhart ring of $\operatorname{HSTAB}(G)$ is different from the ordinary power in general, as the following example shows.

Example 3.11. Let $X:=\left\{x_{i} \mid 0 \leq i \leq 6\right\}, Y:=\left\{y_{i} \mid 0 \leq i \leq 6\right\}, Z:=\left\{z_{i} \mid 0 \leq\right.$ $i \leq 6\}, V:=X \cup Y \cup Z$ and

$$
E:=\left\{\left\{x_{i}, x_{i+1}\right\},\left\{x_{i}, y_{i}\right\},\left\{y_{i}, z_{i}\right\},\left\{z_{i}, y_{i+1}\right\},\left\{z_{i}, z_{i+3}\right\} \mid 0 \leq i \leq 6\right\},
$$

where indices are considered modulo 7 , and set $G:=(V, E)$. 


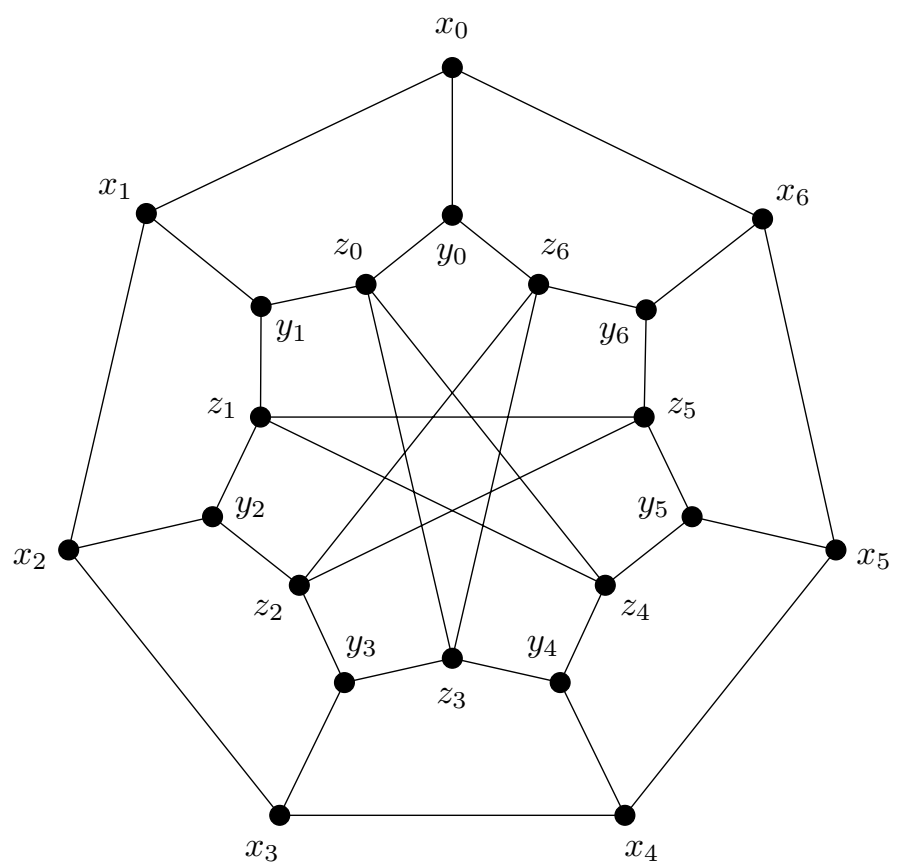

Note that $Y$ is a stable set, $X$ and $Z$ are 7-cycles without chord and no elements of $X$ and $Z$ are adjacent.

We first show that $G$ has no triangle. Assume the contrary and let $T$ be a triangle in $G$. Since no elements of $X$ and $Z$ are adjacent, we see that $T \subset X \cup Y$ or $T \subset Z \cup Y$. Since $X$ and $Z$ are 7 -cycles without chord, $T$ can not be contained in $X$ or $Z$. Further, since $Y$ is a stable set, $T$ can not contain 2 or more elements of $Y$. Thus, $T$ contains exactly 1 element of $Y$. By symmetry, we may assume that $T \cap Y=\left\{y_{0}\right\}$. If $T \subset X \cup Y$, then $T$ contains 2 elements of $X$ which are adjacent to $y_{0}$. However, there is only 1 element of $X$ which is adjacent to $y_{0}$. Thus, $T \subset Z \cup Y$. Since elements of $Z$ adjacent to $y_{0}$ are $z_{0}$ and $z_{6}$, we see that $T=\left\{y_{0}, z_{0}, z_{6}\right\}$. This is a contradiction since $z_{0}$ and $z_{6}$ are not adjacent.

Next we show that for any 5 -cycle $C$ in $G, C \cap X \neq \emptyset$ and $C \cap Z \neq \emptyset$. In particular, $\#(C \cap Y) \geq 2$, since no elements of $X$ and $Z$ are adjacent. Assume the contrary. Then $C \subset X \cup Y$ or $C \subset Z \cup Y$. Since $X$ and $Z$ are 7-cycles without chord, we see that $C \not \subset X$ and $C \not \subset Z$. Thus, $C \cap Y \neq \emptyset$. By symmetry, we may assume that $C \ni y_{0}$. Since $y_{0}$ is adjacent to only 1 element $x_{0}$ in $X \cup Y, C \subset X \cup Y$ is impossible. Thus, $C \subset Z \cup Y$. $y_{0}$ is adjacent to exactly 2 elements $z_{0}$ and $z_{6}$ in $Z \cup Y$. Further, $z_{0}$ is adjacent to $z_{3}, z_{4}$ and $y_{1}$ and $z_{6}$ is adjacent to $z_{2}, z_{3}$ and $y_{6}$ in $Z \cup Y$ except $y_{0}$. Since no elements of $\left\{z_{3}, z_{4}, y_{1}\right\}$ and $\left\{z_{2}, z_{3}, y_{6}\right\}$ are adjacent, we see that this case is also impossible. 
Define $\mu \in \mathbb{Z}^{V^{-}}$by $\mu\left(x_{i}\right)=\mu\left(z_{i}\right)=4, \mu\left(y_{i}\right)=3$ for $0 \leq i \leq 6$ and $\mu(-\infty)=10$. Since $\max _{w \in V} \mu(w)=4$, we see that $\mu^{+}(e) \leq 8=\mu(-\infty)-2$ for any $e \in E$. Let $C$ be an arbitrary 5 -cycle. Since $\#(C \cap Y) \geq 2$, we see that

$$
\mu^{+}(C) \leq 18=\mu(-\infty) \frac{5-1}{2}-2 .
$$

Let $C$ be an arbitrary odd cycle with length at least 7 . Then, since $\max _{w \in V} \mu(w)=$ 4 , we see that

$$
\mu^{+}(C) \leq 4(\# C) \leq 5(\# C)-7=\mu(-\infty) \frac{\# C-1}{2}-2 .
$$

Thus, we see that $\mu \in \mathcal{U}^{(2)}$ and therefore $T^{\mu} \in \omega_{E_{\mathbb{K}}[\operatorname{HSTAB}(G)]}^{(2)}$ by Proposition 3.7.

We deduce a contradiction by assuming that there are $\mu_{1}, \mu_{2} \in \mathcal{U}^{(1)}$ with $\mu=$ $\mu_{1}+\mu_{2}$. We may assume that $\mu_{1}(-\infty) \leq \mu_{2}(-\infty)$.

Since $X$ is a 7 -cycle without chord, we see that

$$
\mu_{i}^{+}(X) \leq \mu_{i}(-\infty) \frac{7-1}{2}-1=3 \mu_{i}(-\infty)-1
$$

for $i=1,2$. Further, since

$$
\mu_{1}^{+}(X)+\mu_{2}^{+}(X)=\mu^{+}(X), \quad \mu_{1}(-\infty)+\mu_{2}(-\infty)=\mu(-\infty)
$$

and

$$
\mu^{+}(X)=28=3 \cdot 10-2=3 \mu(-\infty)-2,
$$

we see that

$$
\mu_{i}^{+}(X)=3 \mu_{i}(-\infty)-1
$$

for $i=1,2$. We see that

$$
\mu_{i}^{+}(Z)=3 \mu_{i}(-\infty)-1
$$

for $i=1,2$ by the same way.

Suppose that $\mu_{1}(-\infty) \leq 2$. Then $\mu_{1}^{+}(e) \geq 2 \geq \mu_{1}(-\infty)$ for any $e \in E$, which contradicts to the clique inequality $\mu_{1}^{+}(e) \leq \mu_{1}(-\infty)-1$. Thus $\mu_{1}(-\infty) \geq 3$.

Suppose that $\mu_{1}(-\infty)=3$. Then since $2 \leq \mu_{1}^{+}(e) \leq \mu_{1}(-\infty)-1$ for any $e \in E$ and $\mu_{1}(v) \geq 1$ for any $v \in V$, we see that $\mu_{1}(v)=1$ for any $v \in V$. Thus,

$$
\mu_{1}^{+}(X)=7<8=3 \mu_{1}(-\infty)-1
$$

This contradicts to the fact shown above.

Next assume that $\mu_{1}(-\infty)=4$. Then for any $e \in E, \mu_{1}^{+}(e) \leq \mu_{1}(-\infty)-1=3$ and therefore $2 \mu_{1}^{+}(X)=\sum_{i=0}^{6}\left(\mu_{1}\left(x_{i}\right)+\mu_{1}\left(x_{i+1}\right)\right) \leq 21$. Thus, $\mu_{1}^{+}(X) \leq 10$, since $\mu_{1}^{+}(X)$ is an integer and

$$
\mu_{1}^{+}(X) \leq 10<11=3 \mu_{1}(-\infty)-1
$$


This is a contradiction again.

Finally, suppose that $\mu_{1}(-\infty)=5$. Then for any $e \in E, \mu_{1}^{+}(e) \leq 4=\mu_{1}(-\infty)-$ 1. Since

$$
2 \mu_{1}^{+}(X)=\sum_{j=0}^{6}\left(\mu_{1}\left(x_{j}\right)+\mu_{1}\left(x_{j+1}\right)\right) \leq 7 \cdot 4=28
$$

and

$$
\mu_{1}^{+}(X)=3 \mu_{1}(-\infty)-1=14,
$$

we see that $\mu_{1}\left(x_{j}\right)+\mu_{1}\left(x_{j+1}\right)=4$ for $0 \leq j \leq 6$. By solving system of these linear equations, we see that $\mu_{1}\left(x_{j}\right)=2$ for $0 \leq j \leq 6$. We also see that $\mu_{1}\left(z_{j}\right)=2$ for $0 \leq j \leq 6$ by the same way.

Consider the 5 -cycle $C_{j}=x_{j} y_{j} z_{j} y_{j+1} x_{j+1} x_{j}$ for $0 \leq j \leq 6$. Since

$$
\mu^{+}\left(C_{j}\right)=18=\mu(-\infty) \frac{\# C_{j}-1}{2}-2,
$$

we see by the same argument as above that $\mu_{i}^{+}\left(C_{j}\right)=9$ for $i=1,2$ and $0 \leq j \leq 6$. Since $\mu_{1}\left(x_{j}\right)=\mu_{1}\left(x_{j+1}\right)=\mu_{1}\left(z_{j}\right)=2, \mu_{1}\left(y_{k}\right) \geq 1$ for $k=j, j+1$, we see that

$$
\mu_{1}\left(y_{j}\right)=1 \quad \text { and } \quad \mu_{1}\left(y_{j+1}\right)=2
$$

or

$$
\mu_{1}\left(y_{j}\right)=2 \quad \text { and } \quad \mu_{1}\left(y_{j+1}\right)=1 .
$$

Suppose that $\mu_{1}\left(y_{0}\right)=1$. Then $\mu_{1}\left(y_{1}\right)=2, \mu_{1}\left(y_{2}\right)=1, \ldots, \mu_{1}\left(y_{6}\right)=1$ and $\mu_{1}\left(y_{0}\right)=2$. This is a contradiction. We also deduce a contradiction if $\mu_{1}\left(y_{0}\right)=2$.

Thus, we see that there are no $\mu_{1}, \mu_{2} \in \mathcal{U}^{(1)}$ with $\mu=\mu_{1}+\mu_{2}$. Therefore $T^{\mu} \notin \omega_{E_{\mathbb{K}}[\operatorname{HSTAB}(G)]}^{2}$ and we see that $\omega_{E_{\mathbb{K}}[\operatorname{HSTAB}(G)]}^{2} \subsetneq \omega_{E_{\mathbb{K}}[\operatorname{HSTAB}(G)]}^{(2)}$.

The graph $G$ in this example is not h-perfect. In fact, let $\nu$ be an element of $\mathbb{Z}^{V}$ with $\nu\left(x_{i}\right)=\nu\left(z_{i}\right)=3 / 7$ and $\nu\left(y_{i}\right)=5 / 14$ for $0 \leq i \leq 6$.

We first show that $\nu \in \operatorname{HSTAB}(G)$. It is clear that $\nu(v) \geq 0$ for any $v \in V$. Since $\max _{v \in V} \nu(v)=3 / 7$, we see that $\nu^{+}(e) \leq 1$ for any $e \in E$. Since $G$ has no triangle, we see that $\nu^{+}(K) \leq 1$ for any clique $K$ in $G$. Let $C$ be an arbitrary odd cycle without chord and length at least 5 . If the length of $C$ is greater than or equals to 7 , then

$$
\nu^{+}(C) \leq \frac{3}{7}(\# C)=\frac{1}{2}(\# C)-\frac{1}{14}(\# C) \leq \frac{1}{2}(\# C)-\frac{1}{14} \cdot 7=\frac{\# C-1}{2} .
$$

If $\# C=5$, then $C$ is one of $C_{i}$ above. Therefore,

$$
\nu^{+}(C)=\nu^{+}\left(C_{i}\right)=3 \cdot \frac{3}{7}+2 \cdot \frac{5}{14}=2=\frac{\# C-1}{2} .
$$

Thus, we see that $\nu \in \operatorname{HSTAB}(G)$. 
Next we show that $\nu \notin \operatorname{STAB}(G)$. Assume the contrary. Then there are stable sets $S_{1}, \ldots, S_{m}$ of $G$ and positive real numbers $\lambda_{1}, \ldots, \lambda_{m}$ such that $\lambda_{1}+\cdots+\lambda_{m}=$ 1 and

$$
\nu=\sum_{j=1}^{m} \lambda_{j} \chi_{S_{j}}
$$

Since $X$ is a 7 -cycle, we see that $\chi_{S_{j}}^{+}(X)=\#\left(S_{j} \cap X\right) \leq 3$ for any $0 \leq j \leq m$. On the other hand, since

$$
3=\nu^{+}(X)=\sum_{j=1}^{m} \lambda_{j} \chi_{S_{j}}^{+}(X) \quad \text { and } \quad \lambda_{1}+\cdots+\lambda_{m}=1
$$

we see that $\chi_{S_{j}}^{+}(X)=3$ for any $0 \leq j \leq m$. We also see that $\chi_{S_{j}}^{+}(Z)=3$ and $\chi_{S_{j}}^{+}\left(C_{i}\right)=2$ for $0 \leq i \leq 6$ and for $0 \leq j \leq m$ by the same way.

Set $S:=S_{1}$. Since $\#(S \cap X)=\chi_{S}^{+}(X)=3$ and $S$ is a stable set, we may assume, by symmetry, that $S \cap X=\left\{x_{0}, x_{2}, x_{4}\right\}$. Consider $S \cap C_{1}$. Since $x_{2} \in S$ and $S$ is a stable set, we see that $y_{2} \notin S$. Thus, $S \cap C_{1}=\left\{x_{2}, y_{1}\right\}$ or $S \cap C_{1}=\left\{x_{2}, z_{1}\right\}$. We see that $S \cap C_{2}=\left\{x_{2}, y_{3}\right\}$ or $S \cap C_{2}=\left\{x_{2}, z_{2}\right\}$ by the same way.

First consider the case where $S \cap C_{1}=\left\{x_{2}, y_{1}\right\}$ and $S \cap C_{2}=\left\{x_{2}, y_{3}\right\}$. Then $S \cap C_{0}=\left\{x_{0}, y_{1}\right\}$ and $S \cap C_{3}=\left\{x_{4}, y_{3}\right\}$ since $\#\left(S \cap C_{0}\right)=\#\left(S \cap C_{3}\right)=2$. Thus, $z_{0}, z_{1}, z_{2}, z_{3} \notin S$. Since $\#(S \cap Z)=3$, we see that $S \cap Z=\left\{z_{4}, z_{5}, z_{6}\right\}$. Since $x_{0}, x_{4} \in S$ and $\#\left(S \cap C_{4}\right)=\#\left(S \cap C_{6}\right)=2$, we see that $S \cap C_{4}=\left\{x_{4}, z_{4}\right\}$ and $S \cap C_{6}=\left\{x_{0}, z_{6}\right\}$. Thus, we see that $x_{5}, y_{5}, x_{6}, y_{6} \notin S$ and therefore, $\#\left(S \cap C_{5}\right)=1$. This contradicts to the fact that $\#\left(S \cap C_{5}\right)=2$.

Next consider the case where $S \cap C_{1}=\left\{x_{2}, y_{1}\right\}$ and $S \cap C_{2}=\left\{x_{2}, z_{2}\right\}$. Since $Z$ is a 7-cycle $z_{0} z_{3} z_{6} z_{2} z_{5} z_{1} z_{4} z_{0}, z_{1} \notin S, z_{2} \in S, \#(S \cap Z)=3$ and $S$ is a stable set, we see that $S \cap Z=\left\{z_{2}, z_{3}, z_{4}\right\}$. Since $x_{4}, z_{4} \in S \cap C_{4}$ and $\#\left(S \cap C_{4}\right)=2$, we see that $S \cap C_{4}=\left\{x_{4}, z_{4}\right\}$. Therefore, $y_{5} \notin S$. Further, $x_{5}, x_{6} \notin S$ by our assumption and $z_{5} \notin S$ by the fact shown above. Therefore, $S \cap C_{5} \subset\left\{y_{6}\right\}$, which contradicts to $\#\left(S \cap C_{5}\right)=2$.

The case where $S \cap C_{1}=\left\{x_{2}, z_{1}\right\}$ and $S \cap C_{2}=\left\{x_{2}, y_{3}\right\}$ can not occur by the same reason.

Finally, assume that $S \cap C_{1}=\left\{x_{2}, z_{1}\right\}$ and $S \cap C_{2}=\left\{x_{2}, z_{2}\right\}$. Then $x_{1}, y_{1} \notin S$ and since $y_{0}$ is adjacent to $x_{0} \in S$, we see that $y_{0} \notin S$. Therefore, $S \cap C_{0}=\left\{x_{0}, z_{0}\right\}$ since $\#\left(S \cap C_{0}\right)=2$. We see that $S \cap C_{3}=\left\{x_{4}, z_{3}\right\}$ by the same way. This contradicts to the fact that $S$ is a stable set since $z_{0}$ and $z_{3}$ are adjacent.

Thus, we see that $\nu \notin \operatorname{STAB}(G)$ and therefore, $\operatorname{STAB}(G) \subsetneq \operatorname{HSTAB}(G)$. 


\section{References}

[1] W. Bruns and J. Herzog, Cohen-Macaulay Rings, Cambridge Studies in Advanced Mathematics, 39, Cambridge University Press, Cambridge, 1993.

[2] V. Chvátal, On certain polytopes associated with graphs, J. Combinatorial Theory Ser. B 18(2), (1975), 138-154.

[3] R. M. Fossum, The Divisor Class Group of a Krull Domain, Ergebnisse der Mathematik und ihrer Grenzgebiete, Band 74, Springer-Verlag, New YorkHeidelberg, 1973.

[4] J. Herzog, T. Hibi and D. I. Stamate, The trace of the canonical module, Israel J. Math., 233(1) (2019), 133-165.

[5] T. Hibi and A. Tsuchiya, Odd cycles and Hilbert functions of their toric rings, Mathematics, 8(1) (2020), 22.

[6] M. Hochster, Rings of invariants of tori, Cohen-Macaulay rings generated by monomials and polytopes, Ann. of Math., 96 (1972), 318-337.

[7] M. Miyazaki, On the canonical ideal of the Ehrhart ring of the chain polytope of a poset, J. Algebra, 541 (2020), 1-34.

[8] H. Ohsugi and T. Hibi, Special simplices and Gorenstein toric rings, J. Combin. Theory Ser. A, 113(4) (2006), 718-725.

[9] N. Sbihi and J.-P. Uhry, A class of h-perfect graphs, Discrete Math., 51(2) (1984), 191-205.

[10] R. P. Stanley, Hilbert functions of graded algebras, Advances in Math., 28(1) (1978), 57-83.

[11] R. P. Stanley, Two poset polytopes, Discrete Comput. Geom., 1(1) (1986), 9-23.

\section{Mitsuhiro Miyazaki}

Department of Mathematics

Kyoto University of Education

1 Fujinomori, Fukakusa, Fushimi-ku, Kyoto, 612-8522, Japan

e-mail: g53448@kyokyo-u.ac.jp 